\title{
Editorial to the Topical Collection: Comets: Post 67P/Churyumov-Gerasimenko Perspectives
}

\author{
Nicolas Thomas ${ }^{1}$ - Björn J.R. Davidsson ${ }^{2}$ - Laurent Jorda ${ }^{3}$ - Ekkehard Kührt ${ }^{4}$. \\ Raphael Marschall $^{5}$. Colin Snodgrass ${ }^{6} \cdot$ Rafael Rodrigo $^{7,8}$
}

Published online: 7 September 2020

(C) Springer Nature B.V. 2020

The Rosetta mission to comet 67P/Churyumov-Gerasimenko was one of the major highlights in space science over the past decade. Orbiting a cometary nucleus for 2 years and monitoring its activity was a remarkable achievement after more than 20 years of work. The experiment suite onboard Rosetta and its lander element, Philae, was designed to make a detailed analysis of the nucleus and the innermost coma. Given that our knowledge of comets at the time of selection and implementation was restricted to data acquired during fast fly-bys, it was inevitable that the mission would produce both remarkable new scientific results but also would provide data analysis challenges and reveal inadequacies in our initial concept for the mission.

While many of the early publications from Rosetta focused on the results from individual instruments, it was clear that the Rosetta/Philae suite could provide a great deal of new

Comets: Post 67P / Churyumov-Gerasimenko Perspectives

Edited by Nicolas Thomas, Björn Davidsson, Laurent Jorda, Ekkehard Kührt, Raphael Marschall, Colin Snodgrass and Rafael Rodrigo

N. Thomas

1 Space Research and Planetology Division, Physikalisches Inst., University of Bern, Bern, Switzerland

2 Jet Propulsion Laboratory, California Institute of Technology, M/S 183-401, 4800 Oak Grove Drive, Pasadena, CA, USA

3 Laboratoire d'Astrophysique de Marseille, 38 rue Frédéric Joliot-Curie, Marseille, France

4 Deutsches Zentrum für Luft- und Raumfahrt (DLR), Institut für optische Sensorsysteme, Rutherfordstraße 2, Berlin, Germany

5 Department of Space Studies, Southwest Research Institute, Boulder, CO, USA

6 Institute for Astronomy, Royal Observatory, University of Edinburgh, Edinburgh, UK

7 International Space Sciences Institute, Hallerstrasse 5, Bern, Switzerland

8 Centro de Astrobiología (INTA-CSIC), European Space Astronomy Centre (ESAC), Camino Bajo del Castillo s/n, Urb. Villafranca del Castillo, 28692 Villanueva de la Cañada, Madrid, Spain 
science by exploiting synergies between the data products of different instruments. With this in mind, a proposal was made to the EU Horizon 2020 programme called MiARD (Multiinstrument Analysis of Rosetta Data), which aimed at pulling several data sets together to constrain the physical processes evident on the nucleus and in the coma. For programmatic reasons, it was necessary to start the MiARD programme earlier than was perhaps ideal with the instrument teams still actively calibrating and publishing. Nonetheless, the MiARD project produced a series of high quality papers that exceeded its target.

The final element of MiARD was the preparation and execution of a workshop that was held at the International Space Sciences Institute (ISSI) in Bern from 15-19 January, 2018. As is customary for ISSI, it extended participation to include a limited number of scientists (up to the limit that could be supported by ISSI) from outside the MiARD project group. This collection of papers is the result of that workshop of the papers presented and of the subsequent discussions.

The aim was to look at several aspects of the nucleus and innermost coma in a multidisciplinary way but also including links to previous observations of comets and taking a look into the implications for future missions. These were itemized as

1. To review the progress made on multi-instrument data analysis. This included

a. Current status of the development of an integrated 3D shape model and its use for mapping properties and scientific measurements to its facets

b. Current status and results from gas dynamics modelling including activity distributions

c. Current status of dust emission and brightness modelling

d. Current status of analysis of surface physical and morphological structure and thermal balance

e. Current understanding of the coma and surface chemical composition

2. To assess and possibly revise current models of nucleus activity (including potential evidence for different mechanisms) and evolution

3. To assess our current understanding of risk from comets (through perturbation of nuclei by non-gravitational forces and particle impact on interplanetary spacecraft).

4. To draw conclusions to the origin and evolution of the planetary system. To re-assess the (extensive) study work performed on future cometary missions, especially for comet nucleus sample return concepts and provide guidelines as to how this work should be updated in the light of Rosetta's results.

It should be noted that the plasma measurements (which are very interesting as illustrated by many papers in recent literature) and studies of coma chemistry (i.e. reaction chemistry) were not addressed.

Many of the more animated discussions surrounded interpretation of Rosetta data when looking at different instrument results. The papers by El-Maarry et al., Filacchone et al., Vincent et al., and Groussin et al. address the nucleus and its properties in a direct and thorough manner. The relationship of the coma to the activity of the nucleus has been discussed in a paper led by one of several young scientists who attended the workshop (see Marschall et al.) while the work on the refractory to ice mass ratio, which produced the most controversy, is presented in a balanced way by Choukroun et al. Some of the controversies remain but significant steps have been taken to resolve them. The discussion of the gas composition by Rubin et al. complements these two works. The influence of activity on orbit perturbations has been presented by Mottola et al. and we note that this effort clearly revealed that there is work still to do in determining the exact orbit of the nucleus from Rosetta data. Weissman et 
al. have presented the implications for studies of the origins of cometary nuclei - a field that has been blown open by the Rosetta observations of the two lobes and the results from New Horizons. Papers by Keller and Kührt and Thomas et al. cover the degree to which Rosetta achieved its goals and what could be achieved by future missions. The former is a critical and possibly provocative assessment while the latter shows how the outstanding questions have arisen and what types of mission could be thought of to address these issues. The work presented, both here and elsewhere, has also shown that we can still extract significant science from the existing data sets and that these results may yet influence our understanding of $67 \mathrm{P}$ and comets in general.

The editors would like to thank the European Union for providing important parts of funding for our MiARD project and the staff at ISSI for their friendly and generous support.

N. Thomas, B.J.R. Davidsson, L. Jorda, E. Kührt,

R. Marschall, C. Snodgrass, R. Rodrigo.

Publisher's Note Springer Nature remains neutral with regard to jurisdictional claims in published maps and institutional affiliations. 